\title{
Upregulation of circular and linear METTL3 and USP3 in colorectal cancer
}

\author{
BILAL ALKHIZZI ${ }^{1}$, MOHAMMAD IMRAN KHAN ${ }^{1,2}$, AYAT AL-GHAFARI ${ }^{1,3,4}$ and HANI CHOUDHRY ${ }^{1,2}$ \\ ${ }^{1}$ Biochemistry Department, Faculty of Science, ${ }^{2}$ Center for Artificial Intelligence in Precision Medicines, \\ King Abdulaziz University, Jeddah 21589; ${ }^{3}$ Scientific Research Center, Dar Al-Hekma University, Jeddah 22246; \\ ${ }^{4}$ Cancer and Mutagenesis Unit, King Fahd Medical Research Centre, King Abdulaziz University, Jeddah 21589, Saudi Arabia
}

Received February 15, 2021; Accepted June 11, 2021

DOI: $10.3892 / 01.2021 .12936$

\begin{abstract}
Several screening methods are currently used to detect colorectal cancer (CRC). However, these are either under-utilized due to their invasive nature or are limited in terms of their diagnostic ability. Numerous reports have investigated messenger and circular RNA as non-invasive biomarkers, but the majority of gene expression studies using RT-qPCR involve critical errors that often lead to irreproducible findings. In the present study, several of these issues were addressed. To the best of our knowledge, this study reports for the first time the upregulation of both the circular and the linear isoform of USP3 and METTL3 in leukocytes from patients with CRC. The linear transcripts of USP3 and METTL3 exhibited 2.3- and 2-fold increases on average in CRC samples $(n=42$ CRC) compared with the respective healthy controls $(n=32)$, whereas their circular isoforms showed 1.6- and 1.7-fold increases, respectively. Moreover, a strong positive correlation was observed between the circular and linear isoforms of USP3 in the CRC cohort $(\mathrm{P}<0.0001)$, but not in the control group $(\mathrm{P}>0.05)$. In addition, the linear USP3 assay had excellent sensitivity (79\%), specificity (75\%), positive predictive value (81\%), negative predictive value $(73 \%)$ and area under the curve (AUC, 0.8534; P-value <0.0001). The circular (AUC, 0.6946; P-value $=0.0043$ ) and linear (AUC, 0.7202; P-value $=0.0012$ ) METTL3 assays also showed potential; however, this was not the case for the circular USP3 assay ( $\mathrm{P}$-value $>0.05$ ). Taken together, this stringent RT-qPCR approach provides evidence for the viability of using circular and linear RNA molecules as disease biomarkers and may help shed light on the regulatory pathways of CRC.
\end{abstract}

Correspondence to: Dr Hani Choudhry, Biochemistry Department, Faculty of Science, King Abdulaziz University, 90A Al-Marsad Street, Jeddah 21589, Saudi Arabia

E-mail: hchoudhry@kau.edu.sa

Key words: circular RNA, methyltransferase-like 3, biomarkers, ubiquitin-specific peptidase 3 , colorectal cancer

\section{Introduction}

Colorectal cancer (CRC) is the fourth most fatal cancer globally, with almost a million annual deaths $(1,2)$. It has the second and third highest incidence rate among cancers in men and women, respectively (2). Early detection offers a survival rate of up to $90 \%$ (3); however, the disease remains virtually asymptomatic until later stages, at which point the survival rate declines severely to $<10 \%(1,3)$. This underscores the great importance of pursuing early biomarkers of CRC.

The current gold standard in CRC screening is colonoscopy $(1,4-6)$. However, despite its proven success, it remains under-utilized (7), probably due to its invasive nature. Several non-invasive biomarkers have been suggested with varying rates of success including fecal immunochemical testing $(1,4)$ and expression of circulating RNA (8). Furthermore, several epigenetic alterations have been implicated in CRC (4). For example, hypermethylation of the septin 9 promoter has been linked to CRC, and early reports suggested moderate sensitivity and specificity (9). However, larger-scale investigations showed that the sensitivity of CRC detection was $<50 \%$ using this method (10). A stool DNA analysis of multiple targets was developed by Cologuard ${ }^{\circledR}$ (Exact Sciences Corporation), and two independent studies validated its excellent specificity and sensitivity related to CRC $(11,12)$. However, both studies found that the sensitivity of the test dropped $<50 \%$ for patients with advanced precancerous lesions.

Circular RNA (circRNA) molecules have emerged as promising disease biomarkers due to their stability and increased half-life compared with their linear counterparts $(13,14)$. The expression of several circRNA targets has been shown to be altered in CRC tissue compared with normal adjacent tissue (13-15). Mechanistically, several biological functions have been reported for circRNA, such as sponging microRNA (16), binding to proteins (17), acting as protein scaffolds (18) and interacting with RNA polymerase II (19).

However, due to a lack of reproducibility, findings obtained using basic research are rarely transferred to clinical practice (20-22). Investigations by Prinz et al (23) and by Begley and Ellis (24) revealed that 92 and $89 \%$ of the surveyed reverse-transcription-quantitative PCR (RT-qPCR) studies could not be reproduced, respectively, even when the experiments were repeated by the same laboratories in which 
the original experiments were conducted. In two separate reports (20,25), Stephen Bustin, the first author of the Minimum Information for Publication of Quantitative Real-Time PCR Experiments (MIQE) guidelines (26), has been extremely critical of the validity of the results from several studies, citing a number of factors that could lead to erroneous results. These factors include a lack of information regarding the PCR conditions and PCR efficiency, as well as dependence on a single reference gene for normalization. In the present study, these issues were addressed using a stringent, more controlled RT-qPCR approach.

The aim of the present study was to investigate whether the altered expression of circRNA molecules in CRC tissue can also be detected in the blood, in order to evaluate their ability to serve as potential non-invasive biomarkers. The literature was scanned for circRNA candidates reported to be deregulated in CRC by two or more independent research groups, four of which were selected for further analysis based on the involvement of their parent genes in CRC. The expression patterns of their linear counterparts were also examined to gain further insight into the regulatory pathways of the genes that encode them. Using this approach, novel findings on the gene expression patterns of leukocytes of CRC patients are reported, which may have potential for use in CRC screening.

\section{Materials and methods}

Patient and control enrollment, sample acquisition and ethical approval. This case-control study was performed on 74 volunteers aged $31-85$ years. The patients with CRC $(n=42$; mean age $\pm \mathrm{SD}=57.2 \pm 12.5$ years) included 29 males and 12 females (one patient with missing data). The healthy control group ( $n=32$; mean age $\pm S D=49.3 \pm 10.5$ years old) consisted of 19 males and 13 females. Patients with CRC were included if they were Saudis with a confirmed diagnosis of CRC at any tumor-node-metastasis (TNM) stage using histopathological and CT scan biopsies. Non-Saudi patients were excluded from the study. The inclusion criteria for the healthy controls were as follows: i) they had to be Saudis; ii) free of any metabolic or chronic diseases, such as hypertension, diabetes mellitus II and other endocrine disorders); and iii) without any family history of CRC or any other tumor during the time of the study. Samples ( $2 \mathrm{ml}$ whole blood) were collected in EDTA tubes from all participants. The participants routinely visited the Day Care Unit of King Abdulaziz University Hospital (Jeddah, Saudi Arabia) (patients with CRC) or the Blood Bank Unit of King Fahad General Hospital (Jeddah, Saudi Arabia) (controls) in the period August 2015-July 2016). The purpose of the research was explained to the participants, from whom written consent was obtained. The Unit of Biomedical Ethics at The Faculty of Medicine, King Abdulaziz University, approved this study (approval no. 261-15).

Selection criteria for candidate circRNA molecules. A literature search was performed in PubMed (https://pubmed.ncbi. nlm.nih.gov/) and PubMed Central (https://www.ncbi.nlm. nih.gov/pmc/) databases for all articles that reported altered expression of circRNAs in CRC tissue [search terms used were: (circRNA OR circular RNA) and (CRC OR colorectal cancer) in the title/abstract fields]. Scanning both the main manuscripts and the supplementary materials, 13 circRNA candidates were found to be reported by at least two independent research groups (Table I) (27-50). From these, four candidates were selected for the purpose of this study, based on the functional implications of their host genes in CRC: ciRS-7 (27-30), circular methyltransferase-like 3 (circMETTL3; 30,31), circular SNF2 histone linker PHD RING helicase (circSHPRH; 32,33) and circular ubiquitin-specific peptidase 3 (circUSP3; 30,34). The linear isoforms of these circRNA candidates were also selected for further analysis, except for ciRS-7, which has no linear counterpart.

RNA extraction and purity. Unless otherwise stated, all centrifugation steps of the RNA extraction protocol were carried out in room temperature. RNA was extracted from leukocytes using QIAamp RNA blood mini kit (Qiagen $\mathrm{GmbH}$; cat. no. 52304) following the manufacturer's instructions. Briefly, $1 \mathrm{ml}$ of whole blood from each sample was mixed with $5 \mathrm{ml}$ buffer EL, incubated for $15 \mathrm{~min}$ on ice and centrifuged at $400 \mathrm{x} \mathrm{g}$ for $10 \mathrm{~min}$ at $4^{\circ} \mathrm{C}$. The supernatant was then discarded, the resulting pellet was resuspended in $2 \mathrm{ml}$ buffer EL, and centrifugation was repeated. The supernatant was discarded, and $600 \mu \mathrm{l}$ RLT buffer supplemented with $1 \%$ $\beta$-mercaptoethanol was added to the pellet. The lysate was added to a QIAshredder column, then mixed with $600 \mu 170 \%$ ethanol. The lysate-ethanol mixture was transferred to a spin column and centrifuged at 8,000 x g for $15 \mathrm{sec}$ (two successive loads to add the whole lysate-ethanol mixture) and the flow-through was discarded. Then, $700 \mu 1$ of buffer RW1 was added, the mixture was centrifuged at $8,000 \mathrm{x}$ g for $15 \mathrm{sec}$, and the flow-through was discarded. This step was repeated with $500 \mu \mathrm{l}$ buffer RPE, after which $500 \mu \mathrm{l}$ buffer RPE was added, and the mixture was centrifuged at $20,000 \mathrm{x}$ g for $3 \mathrm{~min}$. The spin column was transferred to a new collection tube and $50 \mu \mathrm{l}$ RNase-free water was added. After centrifugation at 8,000 x g for $1 \mathrm{~min}$, the eluate was stored at $-20^{\circ} \mathrm{C}$.

cDNA synthesis. A total of $300 \mathrm{ng}$ RNA from each sample was reverse transcribed using random hexamers in $20-\mu 1$ reactions using a High-Capacity cDNA Reverse Transcription kit (Applied Biosystems; cat. no. 4368814) according to the manufacturer's protocol. Briefly, all RNA samples were adjusted to a final concentration of $30 \mathrm{ng} / \mu \mathrm{l}$ and $10 \mu \mathrm{l}$ of each sample was mixed with $2 \mu \mathrm{l} 10 \mathrm{X}$ random hexamers, $0.8 \mu \mathrm{l}$ 25X dNTP mix, $2 \mu 1$ 10X RT buffer, $1 \mu$ l reverse transcriptase, $3.2 \mu 1$ nuclease-free water and $1 \mu \mathrm{l}$ RNase inhibitor (Applied Biosystems; cat. no. N8080119). The thermal cycler for the cDNA synthesis reaction was set for $10 \mathrm{~min}$ at $25^{\circ} \mathrm{C}$, followed by $120 \mathrm{~min}$ at $37^{\circ} \mathrm{C}$ and finally $5 \mathrm{~min}$ at $85^{\circ} \mathrm{C}$ to inactivate the reverse transcriptase.

Primer design. Primers were designed using the PrimerBLAST toolfrom the NationalCenter for Biotechnology Information-US National Library of Medicine (https://www.ncbi.nlm.nih.gov/ tools/primer-blast/index.cgi?LINK_LOC=BlastHome) and purchased from Macrogen. The primers sequences are listed in Table II.

$R T-q P C R$. The resulting cDNA was used for qPCR using the SsoAdvanced ${ }^{\mathrm{TM}}$ Universal SYBR ${ }^{\circledR}$-Green Supermix (Bio-Rad 
Table I. circRNAs that were reported by at least 2 independent groups to be deregulated in CRC.

\begin{tabular}{llc}
\hline circRNA ID & \multicolumn{1}{c}{ Parent Gene } & (Refs.) \\
\hline hsa_circ_0001946 & CDR1AS (ciRS-7) & $(27-30)$ \\
hsa_circ_0000523 & METTL3 & $(30,31)$ \\
hsa_circ0001649 & SHPRH & $(32,33)$ \\
hsa_circ_0002138 & USP3 & $(30,34)$ \\
hsa_circ_0000284 & HIPK3 & $(28,35)$ \\
hsa_circ_0006990 & VAPA & $(36,37)$ \\
hsa_circ_0026344 & ACVRL1 & $(38,39)$ \\
hsa_circ_0000826 & ANKRD12 & $(40,41)$ \\
hsa_circ_0001313 & CCDC66 & $(42,43)$ \\
hsa_circ_0020397 & DOCK1 & $(44,45)$ \\
hsa_circ_0026782 & ITGA7 & $(30,46)$ \\
Hsa_circ_0001821 & PVT1 & $(36,47)$ \\
hsa_circ_0000518 & RPPH1 & $(41,48)$ \\
hsa_circ_0072088 & ZFR & $(49,50)$ \\
\hline
\end{tabular}

$\mathrm{CRC}$, colorectal cancer.

laboratories, Inc.; cat. no. 1725272) in $11-\mu 1$ reaction volumes and final concentrations of $500 \mathrm{nM}$ for the forward and reverse primers. To ensure equal additions of the cDNA template to all assays, the master mix was prepared with the cDNA template. To avoid inter-run variation, all assays for each sample were carried out on the same run. At least one duplicate of each reaction was set up, and all replicates had a Cq standard error of $<1 \mathrm{Cq}$. The PCR cycling protocol included $2 \mathrm{~min}$ at $95^{\circ} \mathrm{C}$, followed by 40 cycles of $95^{\circ} \mathrm{C}$ for $15 \mathrm{sec}$ and $60^{\circ} \mathrm{C}$ for $30 \mathrm{sec}$ (data collection). These cycles were followed by $95^{\circ} \mathrm{C}$ for $30 \mathrm{sec}$, then an incremental rise from 65 to $95^{\circ} \mathrm{C}$, during which data were collected every $5 \mathrm{sec}$ at $0.5^{\circ} \mathrm{C}$ intervals. Efficiency-corrected $\mathrm{Cq}$ values and corrected relative expression $2^{-\Delta \Delta C q}$ method (51) were determined using CFX Manager version 3.1 (Bio-Rad Laboratories, Inc.) and were used for all subsequent analyses. Initially, three reference genes were used for normalization: GAPDH, ribosomal protein lateral stalk subunit P1 (RPLP1) and ribosomal protein L13A (RPL13A) (see Table II for accession nos.). GAPDH was not used in subsequent experiments due to instability in our conditions, as explained in the Results section.

Reference gene stability. Reference gene stability was determined using the 'target stability value' tool in CFX Manager version 3.1 (Bio-Rad Laboratories, Inc.), following the manufacturer' protocol.

PCR efficiency. The PCR efficiency was calculated using the online qPCR Efficiency Calculator tool (Thermo Fisher Scientific, Inc.) (52).

Statistical analysis. Optimal cut-off points for the receiver operating characteristic (ROC) curves were calculated using the web tool easyROC v.1.3.1 (53). Welch's two-tailed t-test, Welch's one-way ANOVA test, ROC curves, area under the curve (AUC), sensitivity, specificity, positive predictive value (PPV), negative predictive value (NPV) and Pearson's correlation coefficient were calculated using the Prism version 9.0.0 software (GraphPad Software, Inc.). As no significant differences were found among the groups by Welch's one-way ANOVA test, post-hoc analysis was not performed.

\section{Results}

Determination of assay efficiency. To ensure that the results of the present study would be consistent over a wide range of concentrations in the aforementioned PCR conditions, with minimal effect of any PCR inhibitors or unspecific reactions, the efficiency of the qPCR assays was first determined. Each assay was performed on five three-fold serial dilutions of cDNA from a representative leukocyte sample, and $\mathrm{Cq}$ values were plotted against the logarithm of the relative concentration of the cDNA templates (Fig. 1). All assays yielded straight lines $\left(R^{2}>0.97\right)$ with efficiencies of $100 \pm 15 \%$ (Table III). These efficiencies were factored into all subsequent $\mathrm{Cq}$ calculations to account for any small variation across concentrations. This approach ensured that PCR assays were optimal and that reproducible results would be obtained regardless of the amount of template used in the reaction.

Initial analysis of circMETTL3, circSHPRH, circUSP3, their linear counterparts and ciRS-7. Welch's two-tailed t-test was used to analyze the expression patterns of circMETTL3, circSHPRH, circUSP 3 and their linear counterparts, as well as ciRS-7 (Table SI) in CRC and normal samples ( $\mathrm{n}=8$ each). The data were normalized to RPLP1, RPL13A and GAPDH. Both linear $(\mathrm{P}=0.002)$ and circular $(\mathrm{P}=0.03)$ isoforms of METTL3 were significantly upregulated (2.2- and 1.7-fold, respectively) in patients with CRC compared with the controls. The linear USP3 was significantly upregulated 2.1 -fold $(\mathrm{P}<0.0001)$; however, its circular isoform only showed a trend towards upregulation (2.2-fold change; $\mathrm{P}=0.11)$. Finally, ciRS-7 and the linear SHPRH were not differentially regulated $(\mathrm{P}=0.54$ and 0.59 , respectively), although circSHPRH was upregulated 1.6-fold $(\mathrm{P}=0.03)$. Based on these findings, circMETTL3, circUSP 3 and their linear counterparts were selected for further analysis in the remainder of the samples $(n=74)$.

circMETTL3, circUSP3 and their linear counterparts are upregulated in CRC. Although GAPDH is commonly used as a reference gene in RT-qPCR studies of cancer, several reports have documented its overexpression in CRC $(54,55)$. To address this issue, the CFX Manager 'target stability value' tool was used to examine the stability of all three selected reference genes in 74 samples. The tool's recommendations for mean $\mathrm{CV}$ and mean $\mathrm{M}$-values for homogeneous samples are $<0.25$ and $<0.5$, respectively. The only combination that met these criteria consisted of RPL13A and RPLP1 (Table IV). The other three possible combinations, all of which included GAPDH, satisfied neither criterion. Therefore, GAPDH is unsuitable as a reference gene in leukocytes from patients with CRC and was consequently removed from subsequent normalization calculations.

Further analysis of a total of 42 CRC patients and 32 controls revealed that circMETTL3, circUSP3, as well as their linear counterparts, were significantly upregulated in leukocytes from patients with CRC (Fig. 2; Table V). The linear 
Table II. Primer sequences for each gene.

\begin{tabular}{|c|c|c|c|c|}
\hline Gene name & Accession no. or circBase ID & Primer & Sequence, 5'-3' & Amplicon length, bp \\
\hline ciRS-7 & hsa_circ_0001946 & $\begin{array}{l}\text { Forward } \\
\text { Reverse }\end{array}$ & $\begin{array}{l}\text { ACCCAGTCTTCCATCAACTGG } \\
\text { GCCATCGGAAACCCTGGATA }\end{array}$ & 112 \\
\hline circMETTL3 & hsa_circ_0000523 & $\begin{array}{l}\text { Forward } \\
\text { Reverse }\end{array}$ & $\begin{array}{l}\text { ACAGAGCAAGAAGATCTACGGA } \\
\text { GAAGCTGTGCTGGGCTTAGG }\end{array}$ & 113 \\
\hline circSHPRH & hsa_circ_0001649 & $\begin{array}{l}\text { Forward } \\
\text { Reverse }\end{array}$ & $\begin{array}{l}\text { CCGAATTGGACAGACAAAACCT } \\
\text { TTCTGACCACAGCTTCCACTT }\end{array}$ & 136 \\
\hline circUSP3 & hsa_circ_0002138 & $\begin{array}{l}\text { Forward } \\
\text { Reverse }\end{array}$ & $\begin{array}{l}\text { CAGGAGCCAAGGGGATAACA } \\
\text { GGTTGGTTAAAGGTACTTGTGCAT }\end{array}$ & 258 \\
\hline linMETTL3 & NM_019852.5 & $\begin{array}{l}\text { Forward } \\
\text { Reverse }\end{array}$ & $\begin{array}{l}\text { TTTTCCGGTTAGCCTTCGGG } \\
\text { TTCCGTAGATCCAAGTGCCC }\end{array}$ & 226 \\
\hline linSHPRH & NM_001042683.3 & $\begin{array}{l}\text { Forward } \\
\text { Reverse }\end{array}$ & $\begin{array}{l}\text { TGGCTCTGAGGAATCGTGTG } \\
\text { GCACAGATTGGGCAAGGTTC }\end{array}$ & 280 \\
\hline linUSP3 & NM_006537.4 & $\begin{array}{l}\text { Forward } \\
\text { Reverse }\end{array}$ & $\begin{array}{l}\text { CCCGGCTAGAAGCGACAC } \\
\text { AGTCAAACAGACCCAAGGGC }\end{array}$ & 229 \\
\hline GAPDH & NM_002046.7 & $\begin{array}{l}\text { Forward } \\
\text { Reverse }\end{array}$ & $\begin{array}{l}\text { TCACCAGGGCTGCTTTTAAC } \\
\text { GATGATCTTGAGGCTGTTGTCA }\end{array}$ & 389 \\
\hline RPLP1 & NM_001003.3 & $\begin{array}{l}\text { Forward } \\
\text { Reverse }\end{array}$ & $\begin{array}{l}\text { GTCCTTCCGAGGAAGCTAAGG } \\
\text { ATTGATCTTATCCTCCGTGACTGT }\end{array}$ & 187 \\
\hline RPL13A & NM_012423.4 & $\begin{array}{l}\text { Forward } \\
\text { Reverse }\end{array}$ & $\begin{array}{l}\text { GCTAAACAGGTACTGCTGGG } \\
\text { AGCCAGGTACTTCAACTTGTTTC }\end{array}$ & 99 \\
\hline
\end{tabular}

circ, circular; lin, linear; hsa, Homo sapiens; METTL3, methyltransferase-like 3; USP3, ubiquitin-specific peptidase 3; RPLP1, ribosomal protein lateral stalk subunit P1; RPL13A, ribosomal protein L13A; SHPRH, SNF2 histone linker PHD RING helicase.

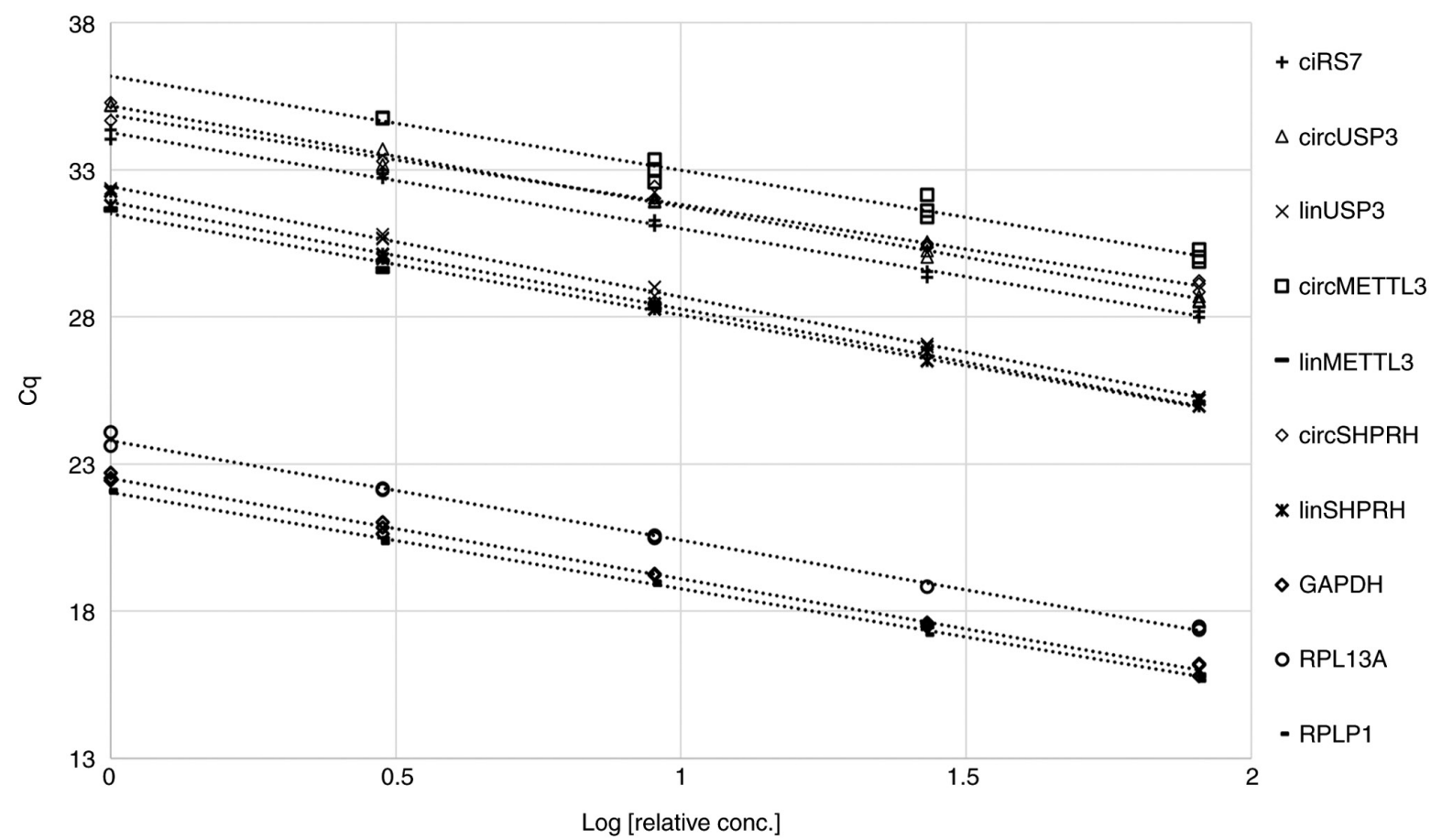

Figure 1. Assay efficiency. Cq values of each assay are plotted against the logarithm of the relative conc. of the starting cDNA template. All assays displayed linearity and efficiency. circ, circular; lin, linear; METTL3, methyltransferase-like 3; USP3, ubiquitin-specific peptidase 3; RPLP1, ribosomal protein lateral stalk subunit P1; RPL13A, ribosomal protein L13A; SHPRH, SNF2 histone linker PHD RING helicase; conc., concentration.

transcript of USP3 had the highest average upregulation, with a 2.3-fold increase $(\mathrm{P}<0.0001)$, while its circular isoform had the lowest upregulation of (1.6-fold; $\mathrm{P}=0.016)$. The expression of the linear transcript of METTL3 nearly doubled on average 
Table III. Assay efficiencies, slopes and $\mathrm{R}^{2}$ values for the trend line of the $\mathrm{Cq}$ vs. logarithm of relative cDNA template concentration plots.

\begin{tabular}{lccc}
\hline Assay & $\mathrm{R}^{2}$ & Slope & Efficiency, \% \\
\hline ciRS-7 & 0.996 & -3.2684 & 102 \\
circMETTL3 & 0.973 & -3.1918 & 106 \\
circSHPRH & 0.985 & -3.0368 & 113 \\
circUSP3 & 0.995 & -3.432 & 96 \\
linMETTL3 & 0.977 & -3.4373 & 95 \\
linSHPRH & 0.995 & -3.6273 & 89 \\
linUSP3 & 0.998 & -3.7538 & 85 \\
GAPDH & 0.997 & -3.4109 & 96 \\
RPL13A & 0.997 & -3.3788 & 98 \\
RPLP1 & 0.998 & -3.2805 & 102 \\
\hline
\end{tabular}

circ, circular; lin, linear; METTL3, methyltransferase-like 3; USP3, ubiquitin-specific peptidase 3; RPLP1, ribosomal protein lateral stalk subunit P1; RPL13A, ribosomal protein L13a; SHPRH, SNF2 histone linker PHD RING helicase.

in $\mathrm{CRC}$ samples $(\mathrm{P}=0.0003)$, and its circular isoform exhibited a 1.7-fold increase $(\mathrm{P}=0.0003)$.

None of the transcripts were differentially regulated based on cancer stage (Welch's one-way ANOVA), sex (Welch's two tailed t-test), or age (Welch's one-way ANOVA, Figs. S1, S2, and S3, respectively).

Correlation between the expression patterns of circular and linear transcripts. To determine whether there was a correlation between circular and linear transcripts of the genes, Pearson's coefficients were calculated between circular and linear isoforms in the CRC and control groups (Fig. 3). There was a strong positive correlation between the circular and linear isoforms of METTL3, both in patients with CRC ( $\mathrm{r}=0.7287$; $\mathrm{P}<0.0001)$ and in healthy controls $(\mathrm{r}=0.7017 ; \mathrm{P}<0.0001)$. Interestingly, while there was no correlation between circular and linear USP3 transcripts in the leukocytes from healthy controls ( $\mathrm{r}=0.3475 ; \mathrm{P}=0.0513)$, a strong positive correlation was observed in patients with $\mathrm{CRC}(\mathrm{r}=0.6788 ; \mathrm{P}<0.0001)$.

Linear USP3 is a potential candidate as a non-invasive $C R C$ biomarker. To determine the diagnostic ability of the candidate transcripts, the AUC was calculated for each assay (Fig. 4, Table VI). The linear USP3 had an AUC of 0.8534 $(\mathrm{P}<0.0001)$ with sensitivity, specificity, PPV and NPV of $79,75,81$ and $73 \%$, respectively. The linear METTL3 assay had excellent sensitivity $(83 \%)$ and moderate PPV and NPV (70 and $71 \%$, respectively), albeit with poor specificity (53.1\%). circMETTL3 and circUSP3 exhibited excellent specificity (94 and 97\%, respectively) and PPV (91 and 92\%, respectively), but had poor sensitivity and NPV $(<50 \%)$.

\section{Discussion}

Suboptimal qPCR assays can lead to erroneous results. Despite the recommendations of the MIQE guidelines (26),
Table IV. CV and mean M-value for each combination of reference genes. Recommendations shown are taken from the Target Stability Value tool in the CFX Manager software.

\begin{tabular}{lcc}
\hline $\begin{array}{l}\text { Reference gene } \\
\text { combination }\end{array}$ & $\begin{array}{c}\text { Mean CV } \\
\text { (recommended } \\
<0.25)\end{array}$ & $\begin{array}{c}\text { Mean M-value } \\
\text { (recommended } \\
<0.5)\end{array}$ \\
\hline RPL13A-RPLP1-GAPDH & 0.3519 & 0.8652 \\
RPL13A-RPLP1 & 0.1597 & 0.4596 \\
RPL13A-GAPDH & 0.3683 & 1.0501 \\
RPLP1-GAPDH & 0.3818 & 1.0858 \\
\hline
\end{tabular}

$\mathrm{CV}$, coefficient of variation; RPLP1, ribosomal protein lateral stalk subunit P1; RPL13A, ribosomal protein L13A.

which are considered the benchmark for RT-qPCR studies, the majority of published articles still fail to report the efficiency of their assays, use one reference gene for normalization and do not clearly report detailed information about their PCR conditions $(20,25)$. To make a stronger claim for the diagnostic ability of our assays, their robustness and reproducibility were ensured by showing evidence of their optimal efficiency and accounted for these efficiencies in the relative expression calculations. Moreover, although three reference genes were initially included, a combination of two reference genes was ultimately used for normalization. The observation that GAPDH was an unsuitable reference gene in the conditions used in this study compounds the impracticality of dependence on a lone reference gene. Moreover, each step taken in the process was described in order to provide complete transparency, which should be an obviously indispensable practice, but is still widely abandoned in the field $(20,25)$. Using this stringent RT-qPCR approach, to the best of our knowledge, the present study reports the first time the upregulation of both the circular and the linear isoform of USP3 and METTL3 in leukocytes from patients with CRC.

All transcripts showed promising diagnostic ability, but the linear isoform of USP3 was remarkable. Its upregulation pattern did not differ based on the available clinicopathological data of the patients, making it a potentially excellent biomarker for detecting CRC at the early stages, when the survival rates are high. Validation of this assay in a larger study cohort is encouraged to confirm its predictive power in cancer and to apply it in a wide range of cancer types to examine whether its upregulation is CRC-specific or common among cancer types.

Despite the observation that circUSP3 is upregulated in leukocytes from patients with CRC, Ruan et al (34) and Bachmayr-Heyda et al (30) reported its downregulation in CRC tissue compared with normal adjacent tissue. The same applies to circMETTL3, which was found to be upregulated in leukocytes from patients with CRC in the present study, but which Jin et al (31) and Bachmayr-Heyda et al (30) identified as downregulated in $12 \mathrm{CRC}$ cell lines and in CRC tissue, respectively. Not much is known about the mechanistic role of circUSP3, although dual luciferase and knockdown/overexpression experiments by Jin et al (31) revealed sponging of 
Table V. Parameters of relative expression between CRC and normal samples.

\begin{tabular}{|c|c|c|c|c|c|}
\hline \multirow[b]{2}{*}{ Assay } & \multicolumn{2}{|c|}{$\begin{array}{l}\text { Mean corrected relative } \\
\text { expression (control) }\end{array}$} & \multirow{2}{*}{$\begin{array}{l}\text { Difference between } \\
\text { means } \pm \text { SEM }\end{array}$} & \multirow{2}{*}{$\begin{array}{l}\text { Average fold } \\
\text { change } \pm \text { SEM }\end{array}$} & \multirow{2}{*}{$\begin{array}{l}\text { Fold change } \\
\text { P-value }\end{array}$} \\
\hline & Control & $\mathrm{CRC}$ & & & \\
\hline circMETTL3 & 0.0017 & 0.0027 & $0.0012 \pm 0.0003$ & $1.730 \pm 0.191$ & 0.0003 \\
\hline linMETTL3 & 0.1456 & 0.2902 & $0.1446 \pm 0.0378$ & $1.993 \pm 0.259$ & 0.0003 \\
\hline circUSP3 & 0.0019 & 0.0031 & $0.0012 \pm 0.0005$ & $1.623 \pm 0.251$ & 0.0158 \\
\hline linUSP3 & 0.1224 & 0.2808 & $0.1584 \pm 0.0335$ & $2.294 \pm 0.274$ & $<0.0001$ \\
\hline
\end{tabular}

CRC, colorectal cancer; circ, circular; lin, linear; METTL3, methyltransferase-like 3; USP3, ubiquitin-specific peptidase 3.
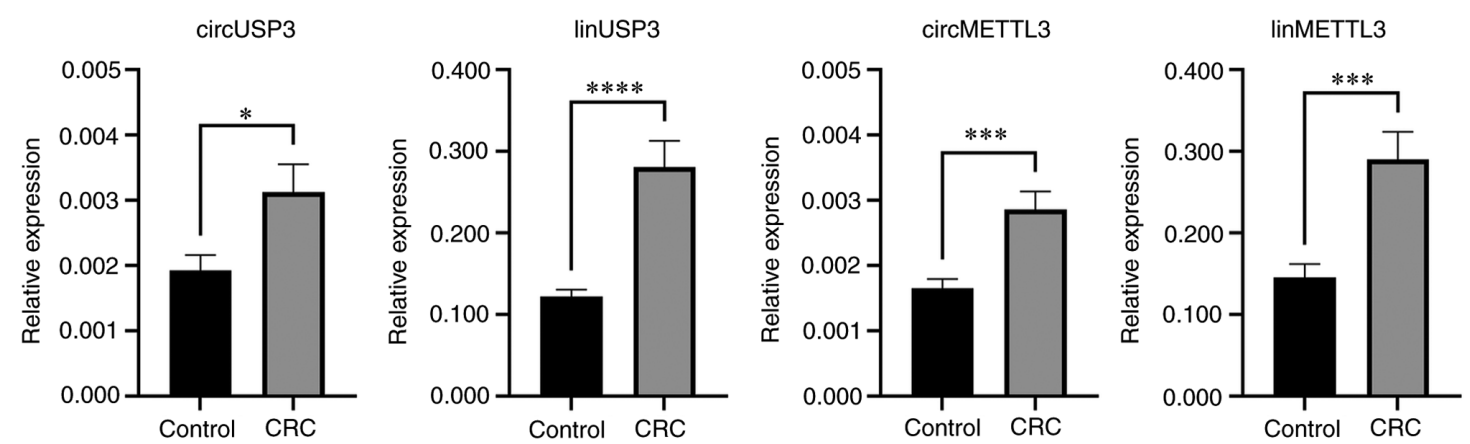

Figure 2. Differential expression in CRC leukocytes. Boxplots representing the relative expression levels of circUSP3, circMETTL3 and their lin counterparts in leukocytes from patients with CRC or healthy controls. All four genes were significantly upregulated. ${ }^{*} \mathrm{P} \leq 0.05,{ }^{* * * *} \mathrm{P} \leq 0.001,{ }^{* * * * *} \mathrm{P} \leq 0.0001$; Welch's two-tailed t-test. Data are presented as the mean \pm SEM. CRC, colorectal cancer; circ, circular; lin, linear; METTL3, methyltransferase-like 3; USP3, ubiquitin-specific peptidase 3 .
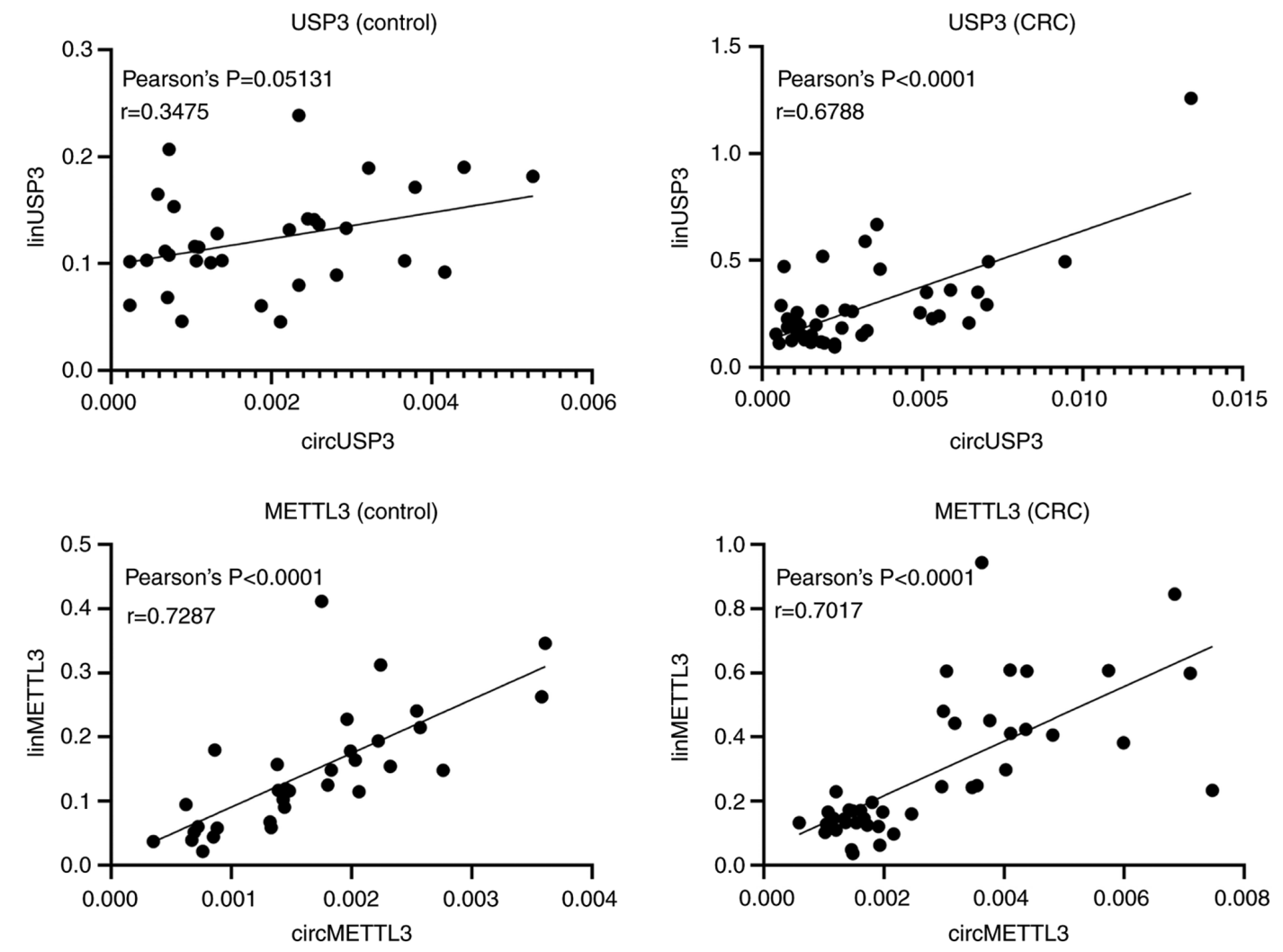

Figure 3. Correlation between circular and linear RNAs in CRC or normal samples. Pearson's correlation analysis of circ and lin transcripts of USP3 and METTL3. Expression levels of circUSP3 and linUSP3 transcripts had a significant positive correlation in the CRC, but not the control group (upper panels). Circular and linear METTL3 had a positive correlation in both CRC and normal samples (lower panel). CRC, colorectal cancer; circ, circular; lin, linear; METTL3, methyltransferase-like 3; USP3, ubiquitin specific peptidase 3. 
Table VI. Receiver operating characteristic analysis of circUSP3, circMETTL3 and their linear counterparts.

\begin{tabular}{lccccccc}
\hline Assay & AUC & P-value & Sensitivity, $\%$ & Specificity, $\%$ & PPV, $\%$ & NPV, \% & $\begin{array}{c}\text { Normalized expression } \\
\text { cut-off }\end{array}$ \\
\hline circMETTL3 & 0.6946 & 0.0043 & 45.2 & 93.8 & 90.5 & 56.6 & $\geq 0.00296$ \\
linMETTL3 & 0.7202 & 0.0012 & 83.3 & 53.1 & 70.0 & 70.8 & $\geq 0.12576$ \\
circUSP3 & 0.6280 & 0.0606 & 26.2 & 96.9 & 91.7 & 50.0 & $\geq 0.00493$ \\
linUSP3 & 0.8534 & $<0.0001$ & 78.6 & 75.0 & 80.5 & 72.7 & $\geq 0.14594$ \\
\hline
\end{tabular}

AUC, area under the curve; PPV, positive predictive value; NPV, negative predictive value; circ, circular; lin, linear; METTL3, methyltransferase-like 3; USP3, ubiquitin-specific peptidase 3.
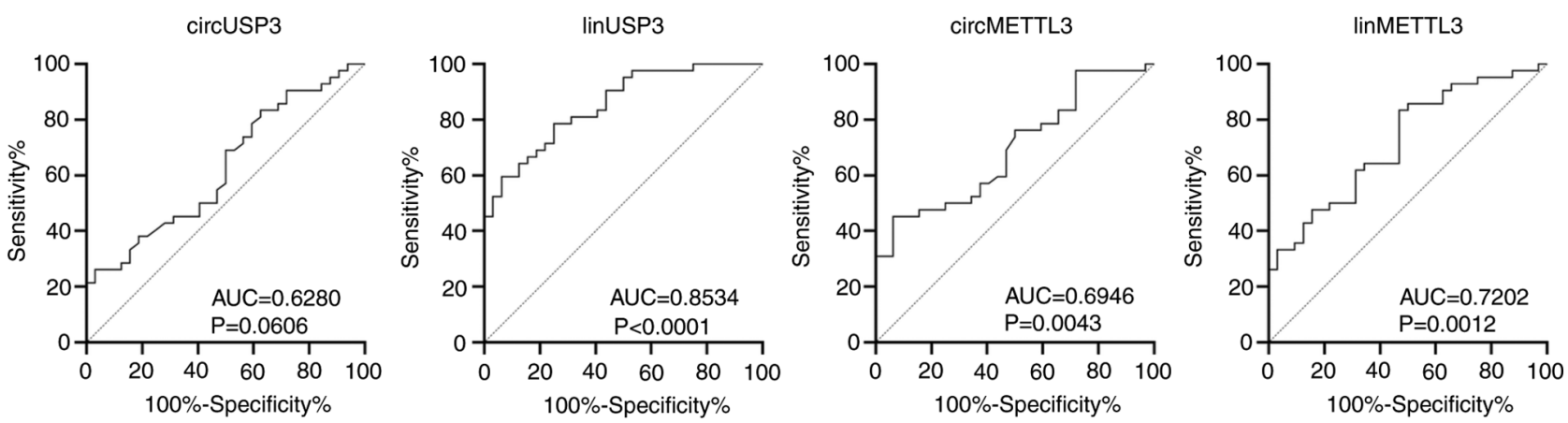

Figure 4. Assay diagnostic performance. Receiver operating characteristic curves for circUSP3, circMETTL3 and their linear counterparts. AUC and P-values are also shown. circ, circular; lin, linear; METTL3, methyltransferase-like 3; USP3, ubiquitin specific peptidase 3.

miR-31 by circMETTL3, leading to the deactivation of the Wnt/ $\beta$-catenin signaling pathway. It may be hypothesized, therefore, that despite activation of $\mathrm{Wnt} / \beta$-catenin signaling in CRC tissue, leukocytes can still deactivate this pathway by overexpressing circMETTL3.

The observed overexpression of the linear transcripts of USP3 and METTL3 in leukocytes from patients with CRC is consistent with their upregulation in CRC tissue (56-63). The linear isoform of USP3 is involved in the DNA damage response and its expression is elevated in a number of solid cancers (56). In an interesting multifaceted investigation, Das et al (56) showed that USP3 promoted cell cycle progression in a number of cancer cell lines by inhibiting ubiquitination of the oncogene CDC25A. The linear isoform of METTL3 expresses the only catalytic unit in the methyltransferase complex. It methylates adenosine residues of RNA at N6 and its levels are elevated in numerous cancers, leading to global hypermethylation (57). Nonetheless, whether the mechanisms of action for USP3 and METTL3 in leukocytes are similar to those in CRC tissue in diseased subjects still needs to be verified by further research.

In conclusion, the present study provides the first evidence of the upregulation of circMETTL3 and circUSP3, along with their linear isoforms, in the leukocytes from patients with CRC. This study has the added strength of avoiding some of the critical errors that can lead to irreproducible RT-qPCR results. These four transcripts may represent good candidates for more extensive studies on their potential involvement in CRC progression, and the linear isoform of USP3 has great prospect as a non-invasive biomarker for CRC.

\section{Acknowledgements}

Not applicable.

\section{Funding}

This research and the article processing charge were funded by the Deanship of Scientific Research at King Abdulaziz University, Jeddah (grant no. G: 284-130-1435).

\section{Availability of data and materials}

The datasets used and/or analyzed during this study are available from the corresponding author on reasonable request.

\section{Authors' contributions}

HC conceptualized the experiments. BA designed the experiments. AAG acquired the samples, and BA and AAG performed the experiments. BA, MIK and HC analyzed the data. BA wrote the manuscript. MIK and $\mathrm{HC}$ revised the manuscript for important intellectual content and managed the project. BA and $\mathrm{HC}$ confirmed the authenticity of all the raw data. All authors have read and approved the manuscript.

\section{Ethics approval and consent to participate}

The purpose of the study was explained to all participants and their written consent was obtained before proceeding. The Unit of Biomedical Ethics at The Faculty of Medicine, 
King Abdulaziz University, approved this study (approval. no. 261-15).

\section{Patient consent for publication}

Not applicable.

\section{Competing interests}

The authors declare that they have no competing interests.

\section{References}

1. Dekker E, Tanis PJ, Vleugels JLA, Kasi PM and Wallace MB: Colorectal cancer. Lancet 394: 1467-1480, 2019.

2. Kuipers EJ, Grady WM, Lieberman D, Seufferlein T, Sung JJ, Boelens PG, van de Velde CJ and Watanabe T: Colorectal cancer. Nat Rev Dis Primers 1: 15065, 2015.

3. Yiu AJ and Yiu CY: Biomarkers in colorectal cancer. Anticancer Res 36: 1093-1102, 2016.

4. Bray C, Bell LN, Liang H, Collins D and Yale SH: Colorectal cancer screening. WMJ 116: 27-33, 2017.

5. Singh H, Nugent Z, Demers AA, Kliewer EV, Mahmud SM and Bernstein CN: The reduction in colorectal cancer mortality after colonoscopy varies by site of the cancer. Gastroenterology 139 1128-1137, 2010.

6. Brenner H, Stock C and Hoffmeister M: Effect of screening sigmoidoscopy and screening colonoscopy on colorectal cancer incidence and mortality: Systematic review and meta-analysis of randomised controlled trials and observational studies. BMJ 348: g2467, 2014.

7. US Preventive Services Task Force; Bibbins-Domingo K, Grossman DC, Curry SJ, Davidson KW, Epling JW Jr, García FA, Gillman MW, Harper DM, Kemper AR, et al: Screening for colorectal cancer: US Preventive Services Task Force recommendation statement. JAMA 315: 2564-2575, 2016.

8. Bosch LJ, Carvalho B, Fijneman RJ, Jimenez CR, Pinedo HM, van Engeland M and Meijer GA: Molecular tests for colorectal cancer screening. Clin Colorectal Cancer 10: 8-23, 2011.

9. Grützmann R, Molnar B, Pilarsky C, Habermann JK, Schlag PM, Saeger HD, Miehlke S, Stolz T, Model F, Roblick UJ, et al: Sensitive detection of colorectal cancer in peripheral blood by septin 9 DNA methylation assay. PLoS One 3: e3759, 2008.

10. Church TR, Wandell M, Lofton-Day C, Mongin SJ, Burger M, Payne SR, Castaños-Vélez E, Blumenstein BA, Rösch T, Osborn N, et al: Prospective evaluation of methylated SEPT9 in plasma for detection of asymptomatic colorectal cancer. Gut 63: 317-325, 2014.

11. Imperiale TF, Ransohoff DF, Itzkowitz SH, Levin TR, Lavin P, Lidgard GP, Ahlquist DA and Berger BM: Multitarget stool DNA testing for colorectal-cancer screening. N Engl J Med 370: 1287-1297, 2014.

12. Redwood DG, Asay ED, Blake ID, Sacco PE, Christensen CM, Sacco FD, Tiesinga JJ, Devens ME, Alberts SR, Mahoney DW, et al: Stool DNA testing for screening detection of colorectal neoplasia in Alaska native people. Mayo Clin Proc 91: 61-70, 2016.

13. Wang $\mathrm{P}$ and $\mathrm{He} \mathrm{X}$ : Current research on circular RNAs associated with colorectal cancer. Scand J Gastroenterol 52: 1203-1210, 2017.

14. Lei B, Tian Z, Fan W and Ni B: Circular RNA: A novel biomarker and therapeutic target for human cancers. Int J Med Sci 16: 292-301, 2019.

15. Ng WL, Mohd Mohidin TB and Shukla K: Functional role of circular RNAs in cancer development and progression. RNA Biol 15: 995-1005, 2018.

16. Hansen TB, Jensen TI, Clausen BH, Bramsen JB, Finsen B, Damgaard CK and Kjems J: Natural RNA circles function as efficient microRNA sponges. Nature 495: 384-388, 2013.

17. Ashwal-Fluss R, Meyer M, Pamudurti NR, Ivanov A, Bartok O, Hanan M, Evantal N, Memczak S, Rajewsky N and Kadener S: circRNA biogenesis competes with pre-mRNA splicing. Mol Cell 56: 55-66, 2014.

18. Du WW, Fang L, Yang W, Wu N, Awan FM, Yang Z and Yang BB: Induction of tumor apoptosis through a circular RNA enhancing Foxo3 activity. Cell Death Differ 24: 357-370, 2017.
19. Zhang Y, Zhang XO, Chen T, Xiang JF, Yin QF, Xing YH, Zhu S, Yang $L$ and Chen LL: Circular intronic long noncoding RNAs. Mol Cell 51: 792-806, 2013.

20. Bustin SA: The reproducibility of biomedical research: Sleepers awake! Biomol Detect Quantif 2: 35-42, 2015.

21. Contopoulos-Ioannidis DG, Ntzani E and Ioannidis JP: Translation of highly promising basic science research into clinical applications. Am J Med 11: 477-484, 2003.

22. Ioannidis JP: Evolution and translation of research findings: From bench to where? PLoS Clin Trials 1: e36, 2006.

23. Prinz F, Schlange T and Asadullah K: Believe it or not: How much can we rely on published data on potential drug targets? Nat Rev Drug Discov 10: 712, 2011.

24. Begley CG and Ellis LM: Drug development: Raise standards for preclinical cancer research. Nature 483: 531-533, 2012.

25. Bustin $\mathrm{S}$ and Nolan T: Talking the talk, but not walking the walk: RT-qPCR as a paradigm for the lack of reproducibility in molecular research. Eur J Clin Invest 47: 756-774, 2017.

26. Bustin SA, Benes V, Garson JA, Hellemans J, Huggett J, Kubista M, Mueller R, Nolan T, Pfaffl MW, Shipley GL, et al: The MIQE guidelines: Minimum information for publication of quantitative real-time PCR experiments. Clin Chem 55: 611-622, 2009.

27. Weng W, Wei Q, Toden S, Yoshida K, Nagasaka T, Fujiwara T, Cai S, Qin H, Ma Y and Goel A: Circular RNA ciRS-7-A promising prognostic biomarker and a potential therapeutic target in colorectal cancer. Clin Cancer Res 23: 3918-3928, 2017.

28. Barbagallo C, Brex D, Caponnetto A, Cirnigliaro M Scalia M, Magnano A, Caltabiano R, Barbagallo D, Biondi A, Cappellani A, et al: LncRNA UCA1, upregulated in CRC biopsies and downregulated in serum exosomes, controls mRNA expression by RNA-RNA interactions. Mol Ther Nucleic Acids 7: 229-241, 2018

29. Tang W, Ji M, He G, Yang L, Niu Z, Jian M, Wei Y, Ren L and $\mathrm{Xu}$ J: Silencing CDR1as inhibits colorectal cancer progression through regulating microRNA-7. Onco Targets Ther 10: 2045-2056, 2017.

30. Bachmayr-Heyda A, Reiner AT, Auer K, Sukhbaatar N, Aust S, Bachleitner-Hofmann T, Mesteri I, Grunt TW, Zeillinger R and Pils D: Correlation of circular RNA abundance with proliferation-exemplified with colorectal and ovarian cancer, idiopathic lung fibrosis, and normal human tissues. Sci Rep 5: 8057,2015

31. Jin Y, Yu LL, Zhang B, Liu CF and Chen Y: Circular RNA hsa_circ_0000523 regulates the proliferation and apoptosis of colorectal cancer cells as miRNA sponge. Braz J Med Biol Res 51: e7811, 2018.

32. Li F, Huang Q, Gong Z, Wang H and Chen J: Diagnostic and prognostic roles of circ-SHPRH for solid cancers: A meta-analysis. Onco Targets Ther 12: 4351-4357, 2019.

33. Ji W, Qiu C, Wang M, Mao N, Wu S and Dai Y: Hsa_circ_0001649: A circular RNA and potential novel biomarker for colorectal cancer. Biochem Biophys Res Commun 497: 122-126, 2018.

34. Ruan H, Deng X, Dong L, Yang D, Xu Y, Peng H and Guan M: Circular RNA circ_0002138 is down-regulated and suppresses cell proliferation in colorectal cancer. Biomed Pharmacother 111: 1022-1028, 2019.

35. Zeng K, Chen X, Xu M, Liu X, Hu X, Xu T, Sun H, Pan Y, He B and Wang S: CircHIPK3 promotes colorectal cancer growth and metastasis by sponging miR-7. Cell Death Dis 9: 417, 2018.

36. Li XN, Wang ZJ, Ye CX, Zhao BC, Li ZL and Yang Y: RNA sequencing reveals the expression profiles of circRNA and indicates that circDDX17 acts as a tumor suppressor in colorectal cancer. J Exp Clin Cancer Res 37: 325, 2018.

37. Li XN, Wang ZJ, Ye CX, Zhao BC, Huang XX and Yang L: Circular RNA circVAPA is up-regulated and exerts oncogenic properties by sponging miR-101 in colorectal cancer. Biomed Pharmacother 112: 108611, 2019.

38. Chen S, Zhang L, Su Y and Zhang X: Screening potential biomarkers for colorectal cancer based on circular RNA chips. Oncol Rep 39: 2499-2512, 2018.

39. Yuan Y, Liu W, Zhang Y, Zhang Y and Sun S: CircRNA circ_0026344 as a prognostic biomarker suppresses colorectal cancer progression via microRNA-21 and microRNA-31. Biochem Biophys Res Commun 503: 870-875, 2018.

40. Zhang Z, Song N, Wang Y, Zhong J, Gu T, Yang L, Shen X, Li Y, Yang X, Liu X, et al: Analysis of differentially expressed circular RNAs for the identification of a coexpression RNA network and signature in colorectal cancer. J Cell Biochem 120: 6409-6419, 2019. 
41. Xu H, Wang C, Song $\mathrm{H}, \mathrm{Xu} \mathrm{Y}$ and Ji G: RNA-Seq profiling of circular RNAs in human colorectal cancer liver metastasis and the potential biomarkers. Mol Cancer 18: 8, 2019.

42. Wang L, Peng X, Lu X, Wei Q, Chen M and Liu L: Inhibition of hsa_circ_0001313 (circCCDC66) induction enhances the radio-sensitivity of colon cancer cells via tumor suppressor miR-338-3p: Effects of cicr_0001313 on colon cancer radio-sensitivity. Pathol Res Pract 215: 689-696, 2019.

43. Hsiao KY, Lin YC, Gupta SK, Chang N, Yen L, Sun HS and Tsai SJ: Noncoding effects of circular RNA CCDC66 promote colon cancer growth and metastasis. Cancer Res 77: 2339-2350, 2017.

44. Zhang XL, Xu LL and Wang F: Hsa_circ 0020397 regulates colorectal cancer cell viability, apoptosis and invasion by promoting the expression of the miR-138 targets TERT and PD-L1. Cell Biol Int 41: 1056-1064, 2017.

45. Zhang R, Xu J,Zhao J and Wang X: Silencing of hsa_circ_0007534 suppresses proliferation and induces apoptosis in colorectal cancer cells. Eur Rev Med Pharmacol Sci 22: 118-126, 2018.

46. Li X, Wang J, Zhang C, Lin C, Zhang J, Zhang W, Zhang W, Lu Y, Zheng L and Li X: Circular RNA circITGA7 inhibits colorectal cancer growth and metastasis by modulating the Ras pathway and upregulating transcription of its host gene ITGA7. J Pathol 246: 166-179, 2018.

47. Wang Z, Su M, Xiang B, Zhao K and Qin B: Circular RNA PVT1 promotes metastasis via miR-145 sponging in CRC. Biochem Biophys Res Commun 512: 716-722, 2019.

48. Zheng X, Chen L, Zhou Y, Wang Q, Zheng Z, Xu B, Wu C, Zhou Q, Hu W, Wu C and Jiang J: A novel protein encoded by a circular RNA circPPP1R12A promotes tumor pathogenesis and metastasis of colon cancer via Hippo-YAP signaling. Mol Cancer 18: 47, 2019.

49. Bian L, Zhi X, Ma L, Zhang J, Chen P, Sun S, Li J, Sun Y and Qin J: Hsa_circRNA_103809 regulated the cell proliferation and migration in colorectal cancer via miR-532-3p/FOXO4 axis. Biochem Biophys Res Commun 505: 346-352, 2018.

50. Zhang P, Zuo Z, Shang W, Wu A, Bi R, Wu J, Li S, Sun X and Jiang L: Identification of differentially expressed circular RNAs in human colorectal cancer. Tumour Biol 39: 1010428317694546, 2017.

51. Livak KJ and Schmittgen TD: Analysis of relative gene expression data using real-time quantitative PCR and the 2(-Delta Delta C(T)) method. Methods 4: 402-408, 2001.
52. ThermoFisher Scientific: qPCR efficiency calculator.https://www. thermofisher.com/uk/en/home/brands/thermo-scientific/molecularbiology/molecular-biology-learning-center/molecular-biologyresource-library/thermo-scientific-web-tools/qpcr-efficiencycalculator.html. Accessed December 23, 2020.

53. Goksuluk D, Korkmaz S, Zararsiz G and Karaağaoğlu AE: easyROC: An interactive web-tool for ROC curve analysis using $\mathrm{R}$ language environment. Contributed Res 8: 213-230, 2016.

54. Zhu Y, Yang C, Weng M, Zhang Y, Yang C, Jin Y, Yang W, He Y, Wu Y,Zhang Y, et al: Identification of TMEM208 and PQLC2 as reference genes for normalizing mRNA expression in colorectal cancer treated with aspirin. Oncotarget 8: 22759-22771, 2017.

55. Guo C, Liu S and Sun MZ: Novel insight into the role of GAPDH playing in tumor. Clin Transl Oncol 15: 167-172, 2013.

56. Das S, Chandrasekaran AP, Suresh B, Haq S, Kang JH, Lee SJ, Kim J, Kim J, Lee S, Kim HH, et al: Genome-scale screening of deubiquitinase subfamily identifies USP3 as a stabilizer of Cdc25A regulating cell cycle in cancer. Cell Death Differ 27: 3004-3020, 2020.

57. Li Y, Ge YZ, Xu L, Xu Z, Dou Q and Jia R: The potential roles of RNA N6-methyladenosine in urological tumors. Front Cell Dev Biol 8: 579919, 2020.

58. Wang H, Xu B and Shi J: N6-methyladenosine METTL3 promotes the breast cancer progression via targeting Bcl-2. Gene 722: 144076, 2020.

59. Wang Q, Geng W, Guo H, Wang Z, Xu K, Chen C and Wang S: Emerging role of RNA methyltransferase METTL3 in gastrointestinal cancer. J Hematol Oncol 13: 57, 2020.

60. Zeng C, Huang W, Li Y and Weng H: Roles of METTL3 in cancer: Mechanisms and therapeutic targeting. J Hematol Oncol 13: 117, 2020

61. Fan L, Chen Z, Wu X, Cai X, Feng S, Lu J, Wang H and Liu N: Ubiquitin-specific protease 3 promotes glioblastoma cell invasion and epithelial-mesenchymal transition via stabilizing snail. Mol Cancer Res 10: 1975-1984, 2019.

62. Fang CL, Lin CC, Chen HK, Hseu YC, Hung ST, Sun DP, Uen YH and Lin KY: Ubiquitin-specific protease 3 overexpression promotes gastric carcinogenesis and is predictive of poor patient prognosis. Cancer Sci 109: 3438-3449, 2018.

63. Liao XH, Wang Y, Zhong B and Zhu SY: USP3 promotes proliferation of non-small cell lung cancer through regulating RBM4. Eur Rev Med Pharmacol Sci 6: 3143-3151, 2020. 